\title{
Cortical and spinal adaptations induced by balance training: correlation between stance stability and corticospinal activation
}

\author{
W. Taube,' M. Gruber,' S. Beck, ${ }^{2,3}$ M. Faist, ${ }^{2}$ A. Gollhofer' and M. Schubert ${ }^{2}$ \\ 1 Department of Sport Science, University of Freiburg, Freiburg, Germany \\ 2 Department of Neurology and Clinical Neurophysiology, Freiburg, Germany \\ 3 Human Motor Control Section, National Institute of Neurological Disorders and Stroke, National Institute of Health, Bethesda, MD, \\ USA
}

Correspondence: W. Taube, University of Freiburg, Department of Sport Science. Schwarzwaldstr. 175, 79117 Freiburg i.Br., Gemany. E-mail: wolfgang.taube@sport.unifreiburg.de

\begin{abstract}
Aim: To determine the sites of adaptation responsible for improved stance stability after balance (=sensorimotor) training, changes in corticospinal and spinal excitability were investigated in 23 healthy subjects.

Methods: Neural adaptations were assessed by means of H-reflex stimulation, transcranial magnetic stimulation (TMS) and conditioning of the H-reflex by TMS ( $H_{\text {cond }}$ ) before and after 4 weeks of balance training. All measurements were performed during stance perturbation on a treadmill. Fast posterior translations induced short- (SLR), medium- and long-latency responses (LLR) in the soleus muscle. Motor-evoked potential- (MEP) and

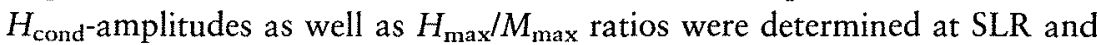
LLR. Postural stability was measured during perturbation on the treadmill. Results: Balance training improved postural stability. $H_{\max } / M_{\max }$ ratios were significantly decreased at LLR. MEPs and $H_{\text {cond }}$ revealed significantly reduced facilitation at LLR following training. A negative correlation between adaptations of $H_{\text {cond }}$ and changes in stance stability was observed $(r=-0.87 ; P<0.01)$ while no correlation was found between stance stability and changes in $H_{\max } / M_{\max }$ ratio. No changes in any parameter occurred at the spinally organized SLR and in the control group.

Conclusion: The decrease in MEP- and $\mathrm{H}_{\text {cond }}$-facilitation implies reduced corticospinal and cortical excitability at the transcortically mediated LLR. Changes in cortical excitability were directly related to improvements in stance stability as shown by correlation of these parameters. The absence of such a correlation between $H_{\max } / M_{\max }$ ratios and stance stability suggests that mainly supraspinal adaptations contributed to improved balance performance following training.

Keywords H-reflex, neuroplasticity, posture, sensorimotor training, transcranial magnetic stimulation.
\end{abstract}

The success of preventive, rehabilitative and physical training interventions largely relies on neural plasticity. Accordingly, knowledge about activity-induced plasticity of the neuromuscular system is crucial for the development and evaluation of training regimens. Balance training [sensorimotor training (SMT)] is frequently used in prevention and rehabilitation of the lower leg because it was shown to restore neuromuscular function following injury as well as prevent ligament sprains and joint injuries (Bahr et al. 1997, Fitzgerald et al. 2000, Verhagen et al. 2004). Furthermore, balance training improved stance stability (Gauffin et al. 1988). 
It is well known that changes of spinal reflex circuits may be related to skill-acquisition and improvements in motor efficiency (for review Wolpaw \& Tennissen 2001, Zehr 2002). Numerous studies concentrated on the H-reflex and its behaviour with respect to physical activity. The modulation of the $\mathrm{H}$-reflex was shown to be highly dependent on the training and testing condition. For instance, strength training increased the H-reflex amplitude (Aagaard et al. 2002, Lagerquist et al. 2006) whereas $\mathrm{H}$-reflexes were inhibited after short-term balance training and during balance tasks (Llewellyn et al. 1990, Trimble \& Koceja 1994, 2001, Mynark \& Koceja 2002). Corticospinal or cortical adaptations induced by balance training were not investigated so far. Only few studies concentrated on the cortical reorganization with respect to the lower leg. Perez et al. (2004) demonstrated that 32 min motor skill training of the tibialis anterior (TA) muscle increased motor-evoked potential (MEP) recruitment curves and maximal MEP amplitudes. Liepert et al. (1999) showed that synchronized thumb and foot movements changed cortical representation. These observations indicate that training-induced cortical plasticity is not limited to motor hand areas. However, the results of both studies specify short-term adaptations and therefore, cannot be transferred to long-lasting balance training used in prevention and rehabilitation. So far, there is no direct evidence that long-term balance training of the lower extremity is capable of affecting supraspinal structures. The present study aimed to highlight the site(s) of neural adaptations induced by long-term balance training of the lower extremity. We were especially interested to clarify whether supraspinal reorganization occurs after balance training. Adaptations of the segmental reflex system and of transcortical loops were investigated with emphasis on their functional context. Several studies using peripheral nerve stimulation or transcranial magnetic stimulation (TMS) indicated that training-specific adaptations were only revealed during muscular activity and not at rest (Voigt et al. 1998, Aagaard et al. 2002, Carroll et al. 2002). Furthermore, changes in spinal and corticospinal excitability proved to be highly task specific (Nielsen $e$ t al. 1993, Mynark \& Koceja 1997, Bonnard et al. 2002, Zehr 2002). Accordingly, peripheral electrical stimulation and TMS were applied during a dynamic perturbation task to uncover training- and balance-specific adaptations on the spinal and cortical level. Stimulation took place at two differently organized reflex responses: at the short-latency response (SLR) and at the longlatency response (LLR). We previously showed that during stance perturbation the LLR of the soleus (SOL) muscle is under supraspinal control while SLR and medium-latency response (MLR) are not (Taube et al. 2006). In the present study, neural adaptations of the
SLR (spinal reflex) were compared with adaptations of the LLR (transcortical reflex). It was assumed that modulations of the SLR reflect spinal adaptations as temporal constraints prevent influence from supraspinal centres. For the LLR, adaptations on the spinal and/or supraspinal level could be of relevance.

\section{Materials and methods}

\section{Subjects}

Twenty-three healthy subjects with no history of neurological disorders or injuries of the lower extremity volunteered to participate in this study. The subjects were randomly allocated into a SMT group (five females and eight males; $25 \pm 3$ years, $66 \pm 9 \mathrm{~kg}$ and $174 \pm 9 \mathrm{~cm}$ ) and a control (CON) group (four females and six males; $27 \pm 5$ years, $67 \pm 8 \mathrm{~kg}$ and $176 \pm 8 \mathrm{~cm}$ ). As two subjects of the SMT group were measured with another TMS protocol, TMS results of these subjects were excluded from analysis. In two other subjects (one in each group) TMS caused discomfort and therefore, they were exclusively measured with peripheral nerve stimulation. None of the subjects participated in any other systematic training during the experiment or had previously performed a SMT. All subjects gave written informed consent to the experimental procedure, which was in accordance with the Declaration of Helsinki and approved by the local ethics committee.

\section{Training}

Details of the training intervention have been reported elsewhere (Gruber et al. 2007). In brief, SMT was performed for 4 weeks in a total of 16 sessions which were all surveyed and supervised by the authors of the study. Each training session lasted for $60 \mathrm{~min}$ including a $10 \mathrm{~min}$ warm up and a $10 \mathrm{~min}$ cool down on an ergometer. The SMT consisted of postural stabilization tasks on wobbling boards, spinning tops and different kinds of soft mats and cushions. Each type of exercise contained four sets of $20 \mathrm{~s}$ duration with $20 \mathrm{~s}$ rest in between (increased to six sets after eight sessions). The rest between the exercises was set to $5 \mathrm{~min}$. Subjects had to maintain balance on each of the stabilization devices with their right leg, barefoot, hands akimbo and eyes open. Subjects of the CON group maintained their normal physical activities throughout the experimental period and were measured again 4 weeks after their initial test.

\section{General experimental procedure}

The experiments were conducted before and after the training period. The post-measurement took place at 
least $24 \mathrm{~h}$ after the last training session to avoid fatigue. The subjects stood in upright position with both legs (stance width individually set to $15-19 \mathrm{~cm}$ between heel centres) on a special treadmill (Woodway) which was accelerated with $60 \mathrm{~m} \mathrm{~s}^{-2}$ in posterior direction. The mechanical stimulus had a translatory amplitude of $15 \mathrm{~cm}$ with a rise time of $50 \mathrm{~ms}$. The time interval between perturbations was randomly set between 5 and $6 \mathrm{~s}$. Neurophysiological measurements took place during blocks of $20-40$ posterior perturbations. To minimize anticipatory reactions, translations in forward and backward direction with different magnitudes $(10-15 \mathrm{~cm})$ and varying accelerations $\left(2.8-60 \mathrm{~m} \mathrm{~s}^{-2}\right)$ were randomly performed before each block. Nevertheless, because of the great number of identical perturbations, habituation effects cannot be excluded (Horak et al. 1989, Carpenter et al. 2005).

The fast posterior perturbation evoked several reflex peaks in the SOL electromyogram (EMG). When subjects got accustomed to the testing condition, the reflex latencies were determined with respect to the onset of ankle movement, which was measured by a goniometer. After rectification of the raw EMG, 10 trials were averaged to receive a representative EMG pattern. The first reflex peaks were termed according to their latencies as SLR, MLR and LLR. SLR onset was defined as the first deflection in the EMG, which exceeded the level of the mean background activity during standing by three-times its standard deviation. The onsets and peaks of MLR and LLR were determined on the basis of the SLR onset and previously reported latencies and durations of SLR, MLR and LLR (Petersen et al. 1998a, Sinkjaer et al. 1999, Grey et al. 2001, Kawashima et al. 2004). The time window for the MLR peak was set from 60 to $85 \mathrm{~ms}$ to exclude monosynaptic reflex responses as well as transcortical loops. Petersen et al. (1998a) calculated the minimum conduction time for a transcortical reflex loop as the sum of the latency of the somatosensory-evoked cortical potential and the latency of the MEP. Without considering the time for central processing, the minimum conduction time would be $79 \mathrm{~ms}$. The LLR response in their study had a mean onset latency of $94 \mathrm{~ms}$. Thus, the authors suggested that the delay required for central processing of the afferent input was approx. $15 \mathrm{~ms}$. However, as demonstrated by Toft et al. (1989) muscle contractions reduced the latency of the LLR by $8 \mathrm{~ms}$ on average. Taking this into account Christensen et al. (2000) calculated the conduction time of a transcortical loop to be as short as $86 \mathrm{~ms}$. Therefore, each reflex peak from $86 \mathrm{~ms}$ onwards after the onset of ankle movement was termed LLR. In a previous study, we demonstrated that in some subjects cortical excitability increased as early as $86 \mathrm{~ms}$ following stance perturbation (Taube et al. 2006). Moreover, this excitability increased with increasing time from the onset of perturbation. To ensure cortical contribution to the compensatory muscular response, LLR around $120 \mathrm{~ms}$ were selected in the present study.

At SLR and LLR, electrical nerve stimulation was applied during perturbation to evaluate spinal excitability while corticospinal excitability was tested using TMS. Activity of direct corticospinal pathways was assessed by conditioning of the H-reflex with subthreshold TMS. Thus, after determining latencies of SLR and LLR and after recording $\mathrm{H}$-reflex recruitment curves (see H-reflex recording), each subject was tested in four conditions.

(1) Stance perturbation alone to elicit SLR and LLR, i.e. to determine the background EMG activity.

(2) Stance perturbation + electrical stimulation to monitor the size of the test $\mathrm{H}$-reflex either coinciding with SLR or LLR.

(3) Stance perturbation + subthreshold TMS to assess corticospinal excitability at SLR and LLR peaks.

(4) Stance perturbation + electrical stimulation (=test reflex) + subthreshold TMS (=conditioning stimulus) to assess the contribution of direct (monosynaptic) corticospinal pathways to SLR and LLR peaks.

These four conditions were tested in consecutive trials, which were randomly conducted. Measurements at SLR and LLR were performed during two separate blocks. To avoid systematic effects, half of the subjects started with 'SLR-trials' and the other ones with 'LLRtrials'. Between trials, subjects took a sitting rest to avoid fatigue.

In all conditions, the peaks of the MEP as well as the peaks of the SOL H-reflexes were triggered to coincide with the peaks of SLR and LLR (analogous to Toft $e t$ al. 1989, Petersen et al. 1998a, Christensen et al. 2001; Fig. 1). Subjects underwent approx. 250 perturbations in each test session. They received feedback on their standing position by visualizing the pressure distribution of their feet on a LED display. The LED display operated in steps of $2.5 \mathrm{Nm}$. Thus, subjects had to control pressure distribution of their feet within $10 \mathrm{~N}$ per $25 \mathrm{~cm}$. In each trial, they were asked to return to the initial level. This ensured a constant upright position and similar muscle activation for all trials.

\section{Mechanical recordings}

Efficiency of training to improve balance performance was tested twofold: First, changes in the vertical ground reaction force during stance perturbation were assessed in each trial by force plates underneath the treadmill. The force excursion was analysed by calculating the peak-to-peak amplitudes of the vertical ground reaction 


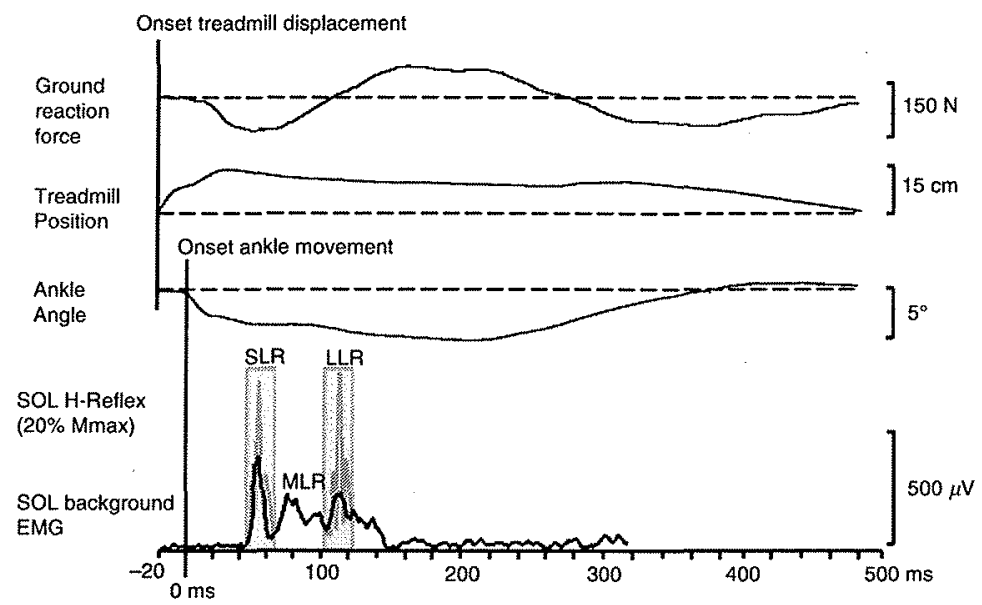

Figure 1 Onset treadmill displacement with corresponding vertical ground reaction force and beginning of ankle movement during perturbed stance on the treadmill. Time zero corresponds to the onset of ankle movement. soleus (SOL) background electromyogram (EMG) (black line) of one subject is displayed. SOL H-reflexes (grey lines) were timed to coincide with the peaks of short- and long-latency response. Background EMG, $\mathrm{H}$-reflexes as well as motor-evoked potentials and conditioned $\mathrm{H}$-reflexes (not illustrated) were compared by analysing root mean square values in a time interval of $20 \mathrm{~ms}$ around each reflex peak (grey boxes).

forces during a time interval of $500 \mathrm{~ms}$ after the onset of perturbation (Fig. 1). Secondly, postural stability was evaluated on a moveable platform (Posturomed ${ }^{\mathrm{TM}}$, Muenchweiler, Germany) capable of producing translational movements in the transversal plane (for technical details see Mueller et al. 2004). During $40 \mathrm{~s}$ standing on the right leg, anterior-posterior and medio-lateral sway paths were recorded by joystick potentiometers connected to the moveable platform. The cumulative sway path of three trials was averaged and values obtained before and after training were compared.

\section{EMG recording}

Bipolar EMG was recorded from TA, gastrocnemius medialis (GM) and SOL muscles of the right leg. After skin preparation $\mathrm{Ag}-\mathrm{AgCl}$ surface cup electrodes $(9 \mathrm{~mm}$ in diameter, centre-to-centre distance $2.5 \mathrm{~cm}$ ) filled with electrode jelly, were firmly attached to the skin in line with the direction of the underlying muscle fibres. EMG signals were sampled at $4 \mathrm{kHz}$, amplified and band-pass filtered $(30-2000 \mathrm{~Hz})$.

\section{$H$-reflex recording}

For H-reflex stimulation, an electrical stimulator (Digitimer ${ }^{\circledR}$ DS 7, Hertfordshire, UK) was used to generate single square-wave pulses of $500 \mu$ s duration. The SOL $\mathrm{H}$-reflex was elicited with the cathode $(2 \mathrm{~cm}$ diameter) placed over the tibial nerve in the popliteal fossa. The anode $(10 \mathrm{~cm} \times 5 \mathrm{~cm})$ was positioned below the patella. H-reflex recruitment curves were recorded during perturbation at SLR and LLR. The electrical stimulation was timed, so that the H-reflex peak coincided with the peaks of the SLR and the LLR component (Fig. 1). Stimuli at SLR and LLR were alternately presented in consecutive trials: During the first perturbation, stimulation took place at SLR during the second perturbation at LLR, etc. At both peaks, $H_{\max } / M_{\max }$ ratios were determined before and after training.

\section{Transcranial magnetic stimulation}

Transcranial magnetic stimulation was applied over the left motor cortex using a Magstim 200 (Magstim Company, Dyfed, UK) with a $90 \mathrm{~mm}$ circular coil. The stimulus waveform was monophasic and had a pulse width of $200 \mu \mathrm{s}$. For each subject the initial stimulation point was set approx. $0.5 \mathrm{~cm}$ anterior to the vertex and over the midline. The optimal position for eliciting MEPs in the TA and SOL with minimal intensity was determined by moving the coil anterior and left from the vertex while MEP size of SOL and TA were monitored on an oscilloscope. This spot was marked on the scalp with a felt pen to control any movement of the coil throughout the tests. During perturbation, the coil was fixed using a special halo vest (Schubert et al. 1997). The coil position was repeatedly checked throughout the experiment. The active motor threshold for the SOL was determined during perturbation at SLR. TMS intensity was adjusted to be just below active motor threshold at SLR (0.9 MT) because supraspinal adaptations were expected to have no influence on this response. TMS was timed so that the peaks of the MEPs arrived in the SOL at the same time as SLR or LLR peaks. Root mean square (RMS) values 
from surface EMGs of the right SOL were calculated for time intervals of $20 \mathrm{~ms}$ around each reflex peak (Fig. 1). At each stimulation point at least 16 trials were recorded with TMS and 16 without magnetic stimulation.

\section{H-reflex conditioned by TMS}

For the conditioning of the H-reflex $\left(H_{\text {cond }}\right)$, subthreshold TMS was applied together with peripheral nerve stimulation during the postural task on the treadmill. Thereby, the effect of transcranial pulses could add up with voluntary drive and thus modulate the SOL H-reflex. The stimulus intensities for the peripheral electrical stimulation and the TMS were kept constant throughout the experiment. The size of the H-reflex was measured as the peak-to-peak amplitude and was expressed as a percentage of $M_{\max }$. It was demonstrated that the susceptibility of the H-reflex to conditioning depends on the size of the control reflex (Crone et al. 1990). Therefore, it was ensured that the test reflex always had the same size of $15-25 \%$ of $M_{\max }$. It was taken care that in all cases the $\mathrm{H}$-reflex was on the ascending part of the $\mathrm{H}$-reflex recruitment curve.

Depending on the time interval between TMS and $\mathrm{H}$-reflex, the activity of specific corticospinal pathways can be assessed. Negative interstimulus intervals (ISIs) indicate that the test $\mathrm{H}$-reflex is released before TMS. The first observable H-reflex facilitation can (at least within the first $0.5-1 \mathrm{~ms}$ after its onset) most likely be attributed to the influence of direct monosynaptic projections from the motor cortex to spinal motoneurons (Nielsen et al. 1993). To detect the onset of this short-latency facilitation ISIs between -8 and $0 \mathrm{~ms}$ were tested in intervals of $1 \mathrm{~ms}$ during dynamic plantar flexion on an ankle ergometer. At each ISI, 16 responses of the control H-reflex (solely electrical nerve stimulation) and the conditioned H-reflex (electrical stimulation plus TMS) were recorded. The conditioned H-reflexes $\left(H_{\text {cond }}\right)$ were expressed in per cent of the control H-reflex and the earliest facilitating ISI was determined for each subject (analogous to Nielsen et al. 1993, Petersen et al. 1998b). To avoid contamination of short-latency facilitation by non-monosynaptic effects, the size of this facilitation was always assessed within the initial millisecond after its onset. The selected ISI, which represented activity in direct corticospinal pathways, was applied in the perturbed stance task. Conditioned and unconditioned stimuli were applied at random with at least $5 s$ rest between trials. Stimulation was timed individually so that both, the peak of the MEP and the H-reflex arrived in time with the peak of the SLR or LLR. For each reflex peak, at least 16 responses were averaged.

\section{Statistics}

$H_{\text {max }} / M_{\max }$ ratios, MEPs and $H_{\text {cond }}$ were analysed by a repeated measures ANOVA procedure with the factors of stimulation point (SLR vs. LLR), time (pre- vs. posttraining measurement) and group (SMT vs. CON) [two (group) $\times$ two (time) $\times$ two (stimulation point)]. In case of significant $F$-values $(P<0.05)$ differences between values at selected points in time or between groups were compared by a paired two-sided test (student's $t$-test). Furthermore, the effect size $(f)$ was calculated following Cohen (1969). Thereby, effect sizes of $0.2,0.5$ and 0.8 point towards small, moderate and large effects, respectively. Correlations between adaptations of $H_{\text {cond }}$ and $H_{\max } / M_{\max }$ ratios with changes in vertical peak ground reaction forces were determined using the Pearson correlation coefficient. SPSS 13.0 software (SPSS Inc., Chicago, IL, USA) was used for statistical analysis. Data are presented as group mean values \pm standard error of the mean.

\section{Results}

\section{Postural stability}

Vertical ground reaction force directed to compensate perturbation revealed significant reductions in the peakto-peak amplitudes following SMT (263 $\mathrm{N} \pm 42$ vs. $229 \mathrm{~N} \pm 25, f=0.50$ and $P<0.05)$. These results were not altered when a shorter time interval was analysed ( $200 \mathrm{~ms}$ instead of $500 \mathrm{~ms}$ ). In contrast, no changes were observed between pre- and post-measurement of the CON group (peak-to-peak amplitudes: $205 \mathrm{~N} \pm 27$ vs. $203 \mathrm{~N} \pm 26 ; f=0.13$ ).

The cumulative sway path on the two-dimensional platform was also reduced following SMT $(-28.6 \% \pm 6.3, f=0.47$ and $P<0.05)$ whereas it remained unchanged in the $C O N$ group $(-4.0 \% \pm 11.5, f=0.33)$.

\section{Reflex latencies and background EMG}

Reflex latencies from the initiation of ankle movement to the onset and to the peak of the reflex response in the SOL are presented in Table 1 . Training affected neither the temporal characteristics nor the size of the reflexive response in the time frames $20 \mathrm{~ms}$ around SLR and LLR when measuring EMG activity of GM, TA and SOL. Furthermore, no RMS changes in a time interval of $200 \mathrm{~ms}$ from the onset of perturbation occurred in SOL and GM muscle. Conversely, TA revealed a significant reduction in the first $200 \mathrm{~ms}$ following SMT $(29 \mu \mathrm{V} \pm 4$ vs. $21 \mu \mathrm{V} \pm 3 ; P<0.05)$. No differences between pre- and post-measurement were observed in the CON group. 


\begin{tabular}{|c|c|c|c|c|c|c|c|}
\hline & \multirow[b]{2}{*}{ Group } & \multicolumn{2}{|c|}{ Peak latency (ms) } & \multicolumn{2}{|c|}{ Onset latency (ms) } & \multicolumn{2}{|c|}{$\mathrm{EMG}$ (RMS in $\mu \mathrm{V})$} \\
\hline & & Pre & Post & Pre & Post & Pre & Post \\
\hline \multirow[t]{2}{*}{ SLR } & SMT & $48 \pm 4$ & $47 \pm 5$ & $40 \pm 3$ & $40 \pm 4$ & $56 \pm 7$ & $57 \pm 11$ \\
\hline & $\mathrm{CON}$ & $51 \pm 5$ & $50 \pm 5$ & $43 \pm 6$ & $41 \pm 4$ & $57 \pm 9$ & $62 \pm 12$ \\
\hline \multirow[t]{2}{*}{ LLR } & SMT & $122 \pm 12$ & $124 \pm 13$ & $106 \pm 13$ & $108 \pm 17$ & $53 \pm 9$ & $62 \pm 10$ \\
\hline & $\mathrm{CON}$ & $124 \pm 11$ & $122 \pm 14$ & $114 \pm 11$ & $112 \pm 9$ & $60 \pm 15$ & $63 \pm 12$ \\
\hline \multirow[t]{2}{*}{ H-reflex } & SMT & $39 \pm 3$ & $39 \pm 2$ & $34 \pm 2$ & $33 \pm 3$ & & \\
\hline & CON & $41 \pm 4$ & $41 \pm 4$ & $34 \pm 5$ & $34 \pm 4$ & & \\
\hline
\end{tabular}

Table I Reflex peak and onset latencies for SLR and LLR with corresponding EMG values (RMS in a time interval of $20 \mathrm{~ms}$ around each peak) before and after training of the SOL

Values are expressed as means \pm standard deviations.

CON, control; EMG, electromyogram; LLR, long-latency response; RMS, root mean square; SLR, short-latency response; SMT, sensorimotor training; SOL, soleus.

\section{$H_{\max } / M_{\max }$ ratios}

In each group, $H_{\max } / M_{\max }$ ratios at SLR were signifcantly smaller than $H_{\max } / M_{\max }$ ratios at LLR in preand post-measurement (Fig. 2). Before training, the SMT group and the CON group neither revealed any differences in the $H_{\max } / M_{\max }$ ratio at SLR nor in the $H_{\max } / M_{\max }$ ratio at LLR (Fig. 2). However, following SMT the $H_{\max } / M_{\max }$ ratio at LLR was significantly reduced (SMT; $f=1.07, P<0.05$ ) whereas controls were not changed (CON; $f=0.28, P=0.2$; Fig. 2). SOL $M_{\max }$ values were not altered in any group in pre- and post-test. Similarly, maximal $\mathrm{M}$-waves of the GM remained unchanged following training
(SLR: $\quad 6.25 \mathrm{mV} \pm 0.53$ vs. $6.23 \mathrm{mV} \pm 0.52$; LLR: $5.96 \mathrm{mV} \pm 0.48$ vs. $5.81 \mathrm{mV} \pm 0.50)$.

\section{Motor-evoked potentials}

Facilitatory effects in pre- and post-measurements were expressed in percentage of the background EMG activity of the corresponding reflex peaks SLR and LLR (analogous to Petersen et al. 1998a, Christensen et al. 2001). In all measurements (pre and post) and in all groups (SMT and CON) TMS facilitated the EMG response signifcantly at LLR whereas SLR was not affected (Fig. 3; see also Taube et al. 2006). However, in nine out of 10 subjects tested with TMS, the facilitatory effects were
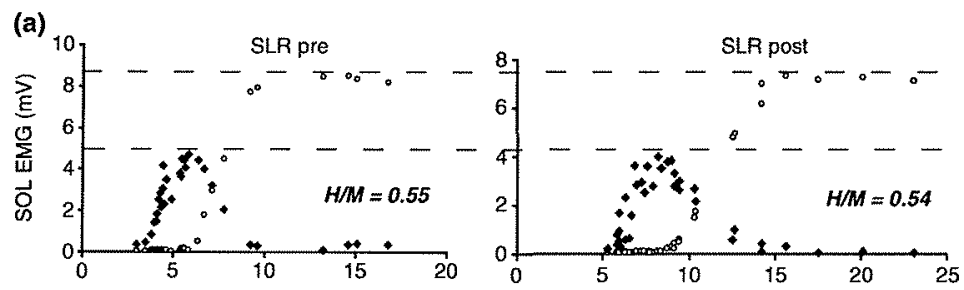

(b)
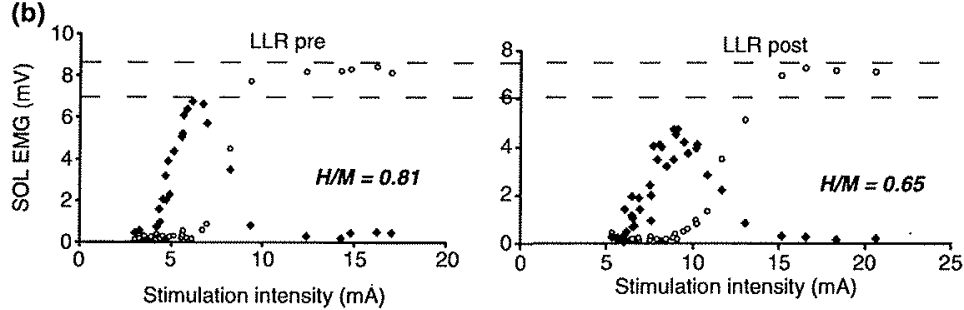

(c)

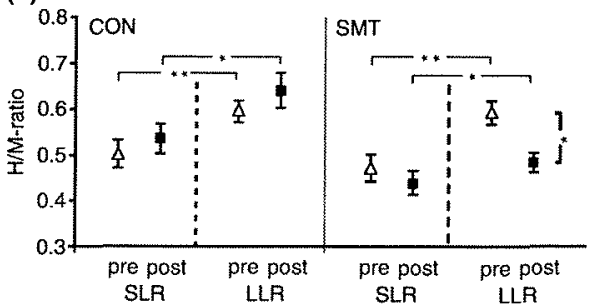

Figure $2 \mathrm{H}$-reflex recruitment curves were recorded during perturbation at short- (SLR) and long-latency response (LLR). In $c$ and $d$, group mean \pm standard error of the mean from pre- and postmeasurement of all subjects of the control and sensorimotor training (SMT) group are displayed. $H_{\max } / M_{\max }$ ratios at SLR were always smaller than the corresponding $H_{\max } / M_{\max }$ ratios at LLR $\left(P<0.05^{*} ; P<0.01^{* *}\right)$. Following SMT, $H_{\text {max }} / M_{\text {max }}$ ratios at LLR were significantly reduced whereas $\mathrm{H}$-reflexes at SLR were not affected. a/b, H-reflex recruitment curves $\bullet \mathrm{H}$-reflexes, $\mathrm{O} \mathrm{M}$-waves) from one subject before and after SMT illustrate that the $H_{\max } / M_{\max }$ ratio was not altered at SLR (a) whereas it was diminished at LLR (b). 
Figure 3 Motor-evoked potentials (MEPs), expressed in percentage of the background electromyogram activity, were recorded at short- (SLR) and longlatency response (LLR) in the soleus during perturbation. MEPs were facilitated at LLR in pre- and post-measurement in both groups $\left(P<0.05^{*} ; P<0.01^{* 4}\right.$ and $\left.P<0.001^{* x^{*}}\right)$. No effects were observed for the SLR. Following sensorimotor training, the facilitation at LLR was significantly reduced.

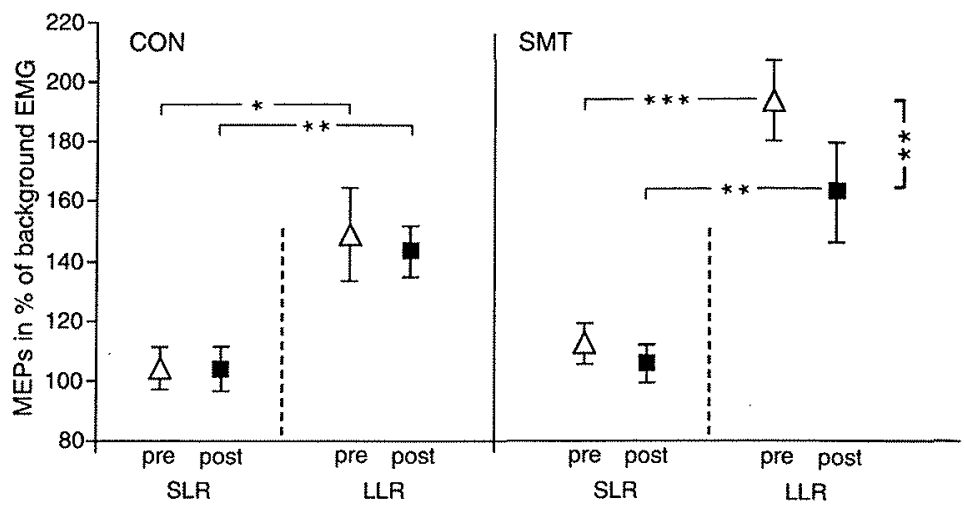

in the post-measurement ( $f=0.33$; Fig. 3 ).

\section{H-reflex conditioned by TMS}

In each group and in pre- and post-measurement the $H_{\text {cond }}$ was significantly facilitated at LLR whereas TMS did not affect the H-reflex at SLR (an example is illustrated in Fig. 4). Subjects of the SMT group significantly reduced their $H_{\text {cond }}$ at LLR following SMT $(144 \% \pm 11$ vs. $118 \% \pm 6, f=0.97$ and $P<0.05$; Fig. $4(f))$. In the CON group, values of preand post-test remained the same size $(122 \pm 8 \%$ vs. $121 \pm 8 \% ; f=0.09$; Fig. 4 (e)). To illustrate, that training effects were not because of alterations in the maximal $M$-wave, changes in the amount of $H$-reflex facilitation were also expressed relative to $M_{\max }$ which was obtained during perturbation at LLR ( $H_{\text {cond }}$ minus control H-reflex as percentage of $M_{\max }$ ). These data also show that facilitation of $H_{\text {cond }}$ was significantly higher before training than after SMT $(6.4 \% \pm 1.6$ vs. $3.3 \% \pm 0.9$ of $M_{\max }, f=0.82$ and $P<0.05$ ).

\section{Correlation of $H_{\text {cond }}$ with change in stance stability}

When correlating training induced adaptations of stance stability on the treadmill (maximal peak amplitudes of the vertical ground reaction force) with alterations of the amount of $\mathrm{H}$-reflex facilitation $\left(H_{\text {cond }}\right)$ a significant interaction of these two parameters was found $(r=$ $0.87 ; P<0.01$; Fig. 5). Subjects who reduced their peak ground reaction force to a great extent, showed also great reductions in the $H_{\text {cond }}$. Conversely, subjects who could not improve their stance stability revealed unchanged $H_{\text {cond }}$ in the post-measurement. In contrast, no such relationship was found between changes in the $H_{\max } / M_{\max }$ ratio and changes in ground reaction force $(r=0.49 ; P=0.2)$. (a)
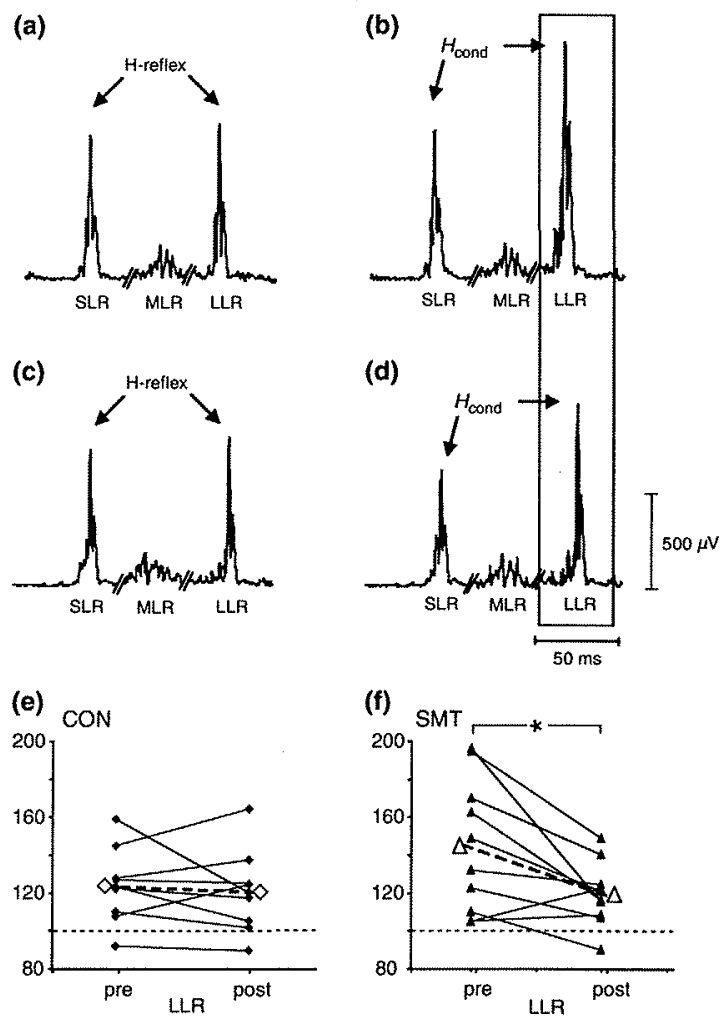

Figure 4 In $a-d$ data from one subject of the sensorimotor training (SMT) group are displayed. H-reflexes were adjusted to $20 \%$ of $M_{\max }$ at short- and long-latency response (LLR) in pre- (a) and post-measurement (c). Conditioning of the H-reflex with subthreshold transcranial magnetic stimulation produced less facilitation at LLR in the post-measurement (d) than before SMT (b). (e) shows facilitation of $H_{\text {cond at LLR in pre- }}$ and post-measurement of all subjects of the control group $H_{\text {cond }}$ were expressed in percentage of the control H-reflex, $\diamond$ corresponds to the mean of all subjects). (f) illustrates changes in the facilitation of $H_{\text {cond }}$ at LLR following SMT ( $\triangle$ mean, $\Delta$ individual values). 


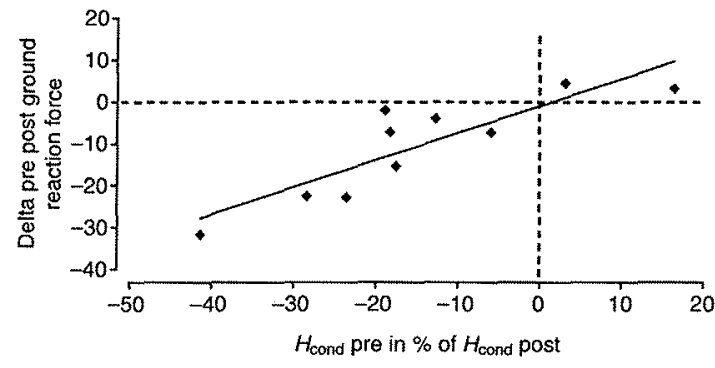

Figure 5 Training-related differences of pre- and post-measurement of the conditioned $\mathrm{H}$-reflex $\left(H_{\text {cond }}\right)$ were illustrated on the abscissa while changes in the vertical peak ground reaction force were displayed on the ordinate. The regression line clearly demonstrates that greater improvements in stance stability (reduced ground reaction forces) were accompanied by greater reductions in the facilitation of $H_{\text {cond }}$.

Similarly to the above-mentioned results, the reduction of the cumulative sway path on the two-dimensional platform showed a tendency to be correlated with changes of $H_{\text {cond }}(r=0.64 ; P=0.062)$ but not with changes of the $H_{\max } / M_{\max }$ ratio.

\section{Discussion}

After balance training, excitability was reduced at spinal, corticospinal and cortical levels when tested during the transcortically mediated LLR. The fact that only changes in cortical excitability were correlated with improved stance stability indicates that supraspinal rather than spinal mechanisms are responsible for the postural improvement.

\section{$H_{\text {max }} / M_{\text {max }}$ ratios}

Following balance training, improved movement control was accompanied by reduced $H_{\max } / M_{\max }$ ratios at LLR. This reduction in spinal excitability is in line with previous training studies showing that improvements in balance led to reduced H-reflexes (Trimble \& Koceja 1994, 2001, Mynark \& Koceja 2002, Schneider \& Capaday 2003). However, in the present study the training induced suppression of the $H_{\text {max }} / M_{\max }$ ratio was only seen when the H-reflex coincided with the LLR whereas it was unaffected when it coincided with the SLR. Changes in background EMG activity did not likely account for this observation as SOL and GM EMG remained unchanged following balance training. One may argue that changes in reciprocal inhibition may play a role. However, TA activity was found to be depressed rather indicating a decrease of reciprocal inhibition. Accordingly, this would suggest increased H-reflexes. The observation of decreased $H_{\max } / M_{\max }$ ratio exclusively at LLR must therefore be explained differently: reduced Ia-transmission may have been responsible, possibly via increased pre-synaptic inhibition (PI). As was shown by Meunier and PierrotDeseilligny (1998), cortical activation of one specific muscle led to reduction of PI when cortical and peripheral Ia-volleys targeted to the same motor nucleus. Furthermore, at the onset of voluntary movements, PI of Ia-afferents is decreased for the contracting muscle; most likely via supraspinal pathways (Hultborn et al. 1987, Iles 1996). As only the LLR is transcortically mediated (Taube $e$ al. 2006) one may expect that PI is generally lower during LLR than during SLR. If this was to be of relevance one would expect that $H_{\max } /$ $M_{\max }$ ratios at LLR were always greater than $H_{\text {max }} l$ $M_{\max }$ ratios at SLR. This was in fact true for all groups (SMT and CON) and in all measurements pre- and post-training (Fig. 2). However, these differences between the $H_{\max } / M_{\max }$ ratios at LLR and SLR were diminished following balance training. Therefore, balance training may have reduced Ia-transmission possibly via increased PI. Alternatively, changes in the $\mathrm{H}$-reflex amplitude following training may be explained by training-induced alterations in body position. Previous work demonstrated the interrelation between H-reflex size and muscle length (Nordlund et al. 2002, Patikas et al. 2004). However, as body position was highly standardized prior to each trial, the compensatory reaction would have to be differently organized in pre- and post-tests within the first $120 \mathrm{~ms}$ to affect muscle length of the SOL. Considering the results of Runge et al. (1999), time constraints probably prevented adaptive reactions in knee and hip joints within this short period of time.

It was assumed that from a functional point of view, inhibition of spinal reflexes in balancing tasks may be advantageous because reflex mediated joint oscillations will be reduced and movement control could be shifted to higher centres (Llewellyn et al. 1990, Aagaard et al. 2002, Solopova et al. 2003). Our results support the view that improved stance stability may - at least partly - be realized by reducing spinal excitability.

\section{Changes in MEP- and $H_{\text {cond }}$ amplitude in relation to balance training}

After balance training, MEPs at LLR were reduced while no changes were observed for the background EMGs and the maximal M-waves of SOL and GM. Alterations in MEP-amplitude reflect changes in corticospinal excitability which could be influenced by modifications on a spinal and/or supraspinal level, i.e. changes in spinal, corticospinal, subcortical or cortical neurons and interneurons, respectively (de Noordhout et al. 1992, Ugawa et al. 1995). Thus, it is not possible from the MEP data to infer the underlying neural mechanisms. Conditioning of the H-reflex by TMS, on 
the other hand, provides a more sophisticated method to explore the adaptations induced by SMT. Here, differences in the spinal excitability were eliminated by adjusting the control $\mathrm{H}$-reflex to $20 \%$ of $M_{\max }$ in preand post-measurements (Nielsen et al. 1993). Moreover, it was unlikely that the magnetically evoked conditioning stimulus was influenced at an interneuron level or by PI provided that the ISI was short enough to exclude non-monosynaptic effects and thus conditioning activated direct, monosynaptic projections. These projections were considered to be free from PI in humans (Rudomin et al. 1975, Nielsen \& Petersen 1994). Therefore, reduced facilitation of $H_{\text {cond }}$ following balance training may either point towards changes in cortical excitability or to changes in the firing rate of the spinal motoneurons and/or their intrinsic firing properties. However, the latter adaptations should have become obvious at SLR, too, as comparable levels of background EMG and similar amplitudes of superimposed $\mathrm{H}$-reflexes existed at SLR and LLR. Furthermore, $H_{\text {max }} / M_{\max }$ ratios were not altered at SLR. Hence, changes in corticomotoneuron excitability were most likely responsible for the reduced $H_{\text {cond }}$ at LLR.

To our knowledge, there exists no TMS study investigating supraspinal (short- or long-term) effects in postural training. Concerning training of the lower extremity in general, cortical/corticospinal plasticity was only investigated after very short training interventions (i.e. 30-45 min Liepert et al. 1999, Perez et al. 2004). Thereby, cortical adaptations occurred following motor skill training and synchronized thumb and foot movements, respectively, but not after non-skill, passive or asynchronous training. Given the fact that exclusively skill training altered corticospinal excitability in both, upper and lower limb, the mechanisms responsible for inducing plastic changes of the leg motor area (Perez et al. 2004) and the hand motor area (PascualLeone et al. 1995) seem similar. Apart from task complexity, the dynamic aspect of the contractions was emphasized: cortical excitability changed in highly dynamic tasks whereas slow contractions did not affect cortical output properties (Muellbacher et al. 2001, Bonato et al. 2002). Balance training involves highly dynamic movements. However, in contrast to the above-mentioned motor skill training studies, muscular activation during balance exercises is not predictable and therefore was not trained in a stereotyped way. Our present results suggest supraspinal adaptations induced by such a variable muscular activity.

\section{How can a reduction of corticospinal excitability be explained?}

In previous studies, decreased corticospinal excitability was shown to occur after fatiguing exercises (McKay et al. 1995, Liepert et al. 1996). In the present study, fatigue is unlikely to account for the depression of MEPs and $H_{\text {cond }}$ because the post-test was accomplished at least $24 \mathrm{~h}$ after the last training session. Furthermore, adaptations concerned only LLR but not SLR which would be expected with fatigue. In addition to that, reduced cortical excitability went along with improved motor performance. This was expressed in: (1) reduced sway path on the free-swinging platform and (2) decreased vertical peak ground reaction forces on the treadmill, which indicated a reduced centre of pressure translation, equivalent to a more stable position of the centre of mass during perturbation. These reductions in peak ground reaction forces were significantly correlated with the reductions of $H_{\text {cond }}$ at LLR indicating that corticospinal activation was reduced when stance stability was increased. Conversely, there was no correlation between changes in the $H_{\max } / M_{\max }$ ratio and changes in vertical peak ground reaction force. Thus, the improvement in balance performance seems to be closely linked to adaptations at the cortical level. Based on previous studies using TMS and functional magnetic resonance imaging it may be deduced that activity of the motor cortex is reduced as soon as the motor task is well trained, i.e. automatic (PascualLeone et al. 1994, Muellbacher et al. 2001, Floyer-Lea \& Matthews 2004, Puttemans et al. 2005). These studies showed that cortical excitability was high in the initial training phase (during skill acquisition) but decreased with progressive training (automatization). It was further demonstrated that while cortical activity decreased, activity of subcortical regions like the basal ganglia and the cerebellum was enhanced (Floyer-Lea \& Matthews 2004, Puttemans et al. 2005). The importance of these structures in automatic (overlearned) motor tasks was highlighted in several previous studies (for review Doyon 1997). Regarding the learning of postural strategies, it was shown that patients with cerebellar deficits showed inability to functional adapt their long-latency reflex gain following changes in the perturbation task (Nashner 1976). As for the present study no intermediate measurements were obtained after the initial training sessions, it can only be speculated that neural control was more and more relegated to subcortical regions with increasing automatization of the task.

\section{Comparison of balance training with long-term motor skill and strength training}

In the present study, 4 weeks of balance training reduced corticospinal and cortical excitability indicated by reduced facilitation of MEPs and $H_{\text {cond }}$ at LLR. The same duration (i.e. 4 weeks) of motor skill training resulted in enhanced maximal MEPs and reduced 
thresholds to elicit MEPs (Jensen et al. 2005). These adaptations were correlated with improvements in motor skill performance and it was therefore concluded that the increased excitability of corticospinal pathways was responsible for the advance in motor behaviour. However, in contrast to the excitability increase in motor skill training tested by Jensen et al. (2005), improved motor performance in our study went along with reduced cortical excitability. Nevertheless, balance training similarly involves supraspinal mechanisms indicated by the correlation of changes in stance stability and cortical excitability. Thus, the supraspinal adaptations occurring in skill-trained and balancetrained subjects seem to differ or at least be different with regard to their temporal appearance. Earlier studies reported strongly time dependent excitability changes (Pascual-Leone et al. 1994, 1999, Muellbacher et al. 2001) which may rely on the type of training.

After strength training, corticospinal excitability. was diminished (Jensen et al. 2005). However, no relationship between changes in strength and changes in corticospinal activity were found. Furthermore, as spinal excitability was not explicitly assessed in this study, it could not be differentiated whether spinal or supraspinal adaptations were responsible for the enhanced strength production after training. Similarly, Carroll et al. (2002) observed reduced corticospinal excitability following 4 weeks of strength training. This reduction was suggested to rely on spinal adaptations. Hence, neither Carroll et al. (2002) nor Jensen et al. (2005) could attribute strength adaptations to supraspinal effects. These considerations imply that adaptations following strength, balance and skill training are specific and may differ because of differences in task complexity, training intensity, movement dynamics and the variability of muscular activity.

\section{Conclusion}

Improved stance stability following balance training was correlated with decreased cortical excitability but not with the changes in spinal excitability. These findings emphasize that cortical plasticity plays a role in the acquisition of balance skills in humans.

\section{Acknowledgement}

This project was supported by the Deutsche Forschungsgemeinschaft (SCHU1487/1-1).

\section{Conflict of interest}

There are no conflicts of interest.

\section{References}

Aagaard, P., Simonsen, E.B., Andersen, J.L., Magnusson, P. \& Dyhre-Poulsen, P. 2002. Neural adaptation to resistance training: changes in evoked $\mathrm{V}$-wave and $\mathrm{H}$-reflex responses. J Appl Pbysiol 92, 2309-2318.

Bahr, R., Lian, O. \& Bahr, I.A. 1997. A twofold reduction in the incidence of acute ankle sprains in volleyball after the introduction of an injury prevention program: a prospective cohort study. Scand J Med Sci Sports 7, 172-177.

Bonato, C., Zanette, G., Fiaschi, A. \& Rossini, P.M. 2002. Activity-dependent modulation of synaptic transmission in the intact human motor cortex revealed with transcranial magnetic stimulation. Cereb Cortex 12, 1057-1062.

Bonnard, M., Camus, M., Coyle, T. \& Pailhous, J. 2002. Taskinduced modulation of motor evoked potentials in upper-leg muscles during human gait: a TMS study. Eur $]$ Neurosci 16, $2225-2230$.

Carpenter, M.G., Thorstensson, A. \& Cresswell, A.G. 2005. Deceleration affects anticipatory and reactive components of triggered postural responses. Exp Brain Res 167, 433-445.

Carroll, T.J., Riek, S. \& Carson, R.G. 2002. The sites of neural adaptation induced by resistance training in humans. J Physiol 544, 641-652.

Christensen, L.O., Petersen, N., Andersen, J.B., Sinkjaer, T. \& Nielsen, J.B. 2000. Evidence for transcortical reflex pathways in the lower limb of man. Prog Neurobiol 62, 251272.

Christensen, L.O., Andersen, J.B., Sinkjaer, T. \& Nielsen, J. 2001. Transcranial magnetic stimulation and stretch reflexes in the tibialis anterior muscle during human walking. J Physiol 531, 545-557.

Cohen, J. 1969. Statistical Power Analysis for the Behavioural Sciences. Academic Press, New York.

Crone, C., Hultborn, H., Mazieres, L., Morin, C., Nielsen, J. \& Pierrot-Deseilligny, E. 1990. Sensitivity of monosynaptic test reflexes to facilitation and inhibition as a funcrion of the test reflex size: a study in man and the cat. Exp Brain Res 81, $35-45$.

Doyon, J. 1997. Skill learning. Int Rev Neurobiol 41, 273-294.

Fitzgerald, G.K., Axe, M.J. \& Snyder-Mackler, L. 2000. The efficacy of perturbation training in nonoperative anterior cruciate ligament rehabilitation programs for physical active individuals. Phys Ther 80, 128-140.

Floyer-Lea, A. \& Matthews, P.M. 2004. Changing brain networks for visuomotor control with increased movement automaticity. I Neuropbysiol 92, 2405-2412.

Gauffin, H., Tropp, H. \& Odenrick, P. 1988. Effect of ankle disk training on postural control in patients with functional instability of the ankle joint. Int J Sports Med 9, 141-144.

Grey, M.J., Ladouceur, M., Andersen, J.B., Nielsen, J.B. \& Sinkjaer, T. 2001. Group II muscle afferents probably contribute to the medium latency soleus stretch reflex during walking in humans. J Physiol 534, 925-933.

Gruber, M., Gruber, S., Taube, W., Schubert, M., Beck, S. \& Gollhofer, A. 2007. Differential effects of ballistic versus sensorimotor training on rate of force development and neural activation in humans. I Strength Cond Res (in press). 
Horak, F.B., Diener, H.C. \& Nashner, L.M. 1989. Influence of central set on human postural responses. J Neurophysiol 62, 841-853.

Hultborn, H., Meunier, S., Pierrot-Deseilligny, E. \& Shindo, M. 1987. Changes in presynaptic inhibition of Ia fibres at the onset of voluntary contraction in man. $J$ Physiol 389 757-772.

Iles, J.F. 1996. Evidence for cutaneous and corticospinal modulation of presynaptic inhibition of la afferents from the human lower limb. J Physiol 491, 197-207.

Jensen, J.L., Marstrand, P.C. \& Nielsen, J.B. 2005. Motor skill training and strength training are associated with different plastic changes in the central nervous system. $J$ Appl Physiol 99, 1558-1568.

Kawashima, N., Nakazawa, K., Yamamoto, S.I., Nozaki, D., Akai, M. \& Yano, H. 2004. Stretch reflex excitability of the anti-gravity ankle extensor muscle in elderly humans. Acta Physiol Scand 180, 99-105.

Lagerquist, O., Zehr, E.P. \& Docherty, D. 2006. Increased spinal reflex excitability is not associated with neural plasticity underlying the cross-education effect. $J$ Appl Physiol 100, 83-90.

Liepert, J., Kotterba, S., Tegenthoff, M. \& Malin, J.P. 1996. Central fatigue assessed by transcranial magnetic stimulation. Muscle Nerve 19, 1429-1434.

Liepert, J., Terborg, C. \& Weiller, C. 1999. Motor plasticity induced by synchronized thumb and foot movements. Exp Brain Res 125, 435-439.

Llewellyn, M., Yang, J.F. \& Prochazka, A. 1990. Human $\mathrm{H}$-reflexes are smaller in difficult beam walking than in normal treadmill walking. Exp Brain Res 83, 22-28.

de Noordhout, M., Pepin, J.L., Gerard, P. \& Delwaide, P.J. 1992. Facilitation of responses to motor cortex stimulation: effects of isometric voluntary contraction. Ann Neurol 32, 365-370.

McKay, W.B., Tuel, S.M., Sherwood, A.M., Stokic, D.S. \& Dimitrijevic, M.R. 1995. Focal depression of cortical excitability induced by fatiguing muscle contraction: a transcranial magnetic stimulation study. Exp Brain Res 105, 276-282.

Meunier, S. \& Pierrot-Deseilligny, E. 1998. Cortical control of presynaptic inhibition of Ia afferents in humans. Exp Brain Res 119, 415-426.

Muellbacher, W., Ziemann, U., Boroojerdi, B., Cohen, L. \& Hallett, M. 2001. Role of the human motor cortex in rapid motor learning. Exp Brain Res 136, 431-438.

Mueller, O., Gunther, M., Krauss, I. \& Horstmann, T. 2004. Physical characterization of the therapeutic device Posturomed as a measuring device - presentation of a procedure to characterize balancing ability. Biomed Tech (Berl) 49, 56-60.

Mynark, R.G. \& Koceja, D.M. 1997. Comparison of soleus H-reflex gain from prone to standing in dancers and controls. Electroencephalogr Clin Neurophysiol 105, 135-140.

Mynark, R.G. \& Koceja, D.M. 2002. Down training of the elderly soleus $\mathrm{H}$ reflex with the use of a spinally induced balance perturbation. J Appl Physiol 93, 127-133.

Nashner, L.M. 1976. Adapting reflexes controlling the human posture. Exp Brain Res 26, 59-72.
Nielsen, J. \& Petersen, N. 1994. Is presynaptic inhibition distributed to corticospinal fibres in man? J Physiol 477 , 47-58.

Nielsen, J., Petersen, N., Deuschl, G. \& Ballegaard, M. 1993. Task-related changes in the effect of magnetic brain stimulation on spinal neurones in man. J Physiol 471, 223-243.

Nordlund, M.M., Thorstensson, A. \& Cresswell, A.G. 2002. Variations in the soleus H-reflex as a function of activation during controlled lengthening and shortening actions. Brain Res 952, 301-307.

Pascual-Leone, A., Grafman, J. \& Hallett, M. 1994. Modulation of cortical motor output maps during development of implicit and explicit knowledge. Science 263, 1287-1289.

Pascual-Leone, A., Nguyet, D., Cohen, L.G., Brasil-Neto, J.P., Cammarota, A. \& Hallett, M. 1995. Modulation of muscle responses evoked by transcranial magnetic stimulation during the acquisition of new fine motor skills. J Neurophysiol 74, 1037-1045.

Pascual-Leone, A., Tarazona, F. \& Catala, M.D. 1999 Applications of transcranial magnetic stimulation in studies on motor learning. Electroencephalogr Clin Neurophysiol Suppl 51, 157-161.

Patikas, D.A., Kotzamanidis, C., Robertson, C.T. \& Koceja, D.M. 2004. The effect of the ankle joint angle in the level of soleus Ia afferent presynaptic inhibition. Electromyogr Clin Neurophysiol 44, 503-511.

Perez, M.A., Lungholt, B.K., Nyborg, K. \& Nielsen, J.B. 2004. Motor skill training induces changes in the excitability of the leg cortical area in healthy humans. Exp Brain Res 159, 197-205.

Petersen, N., Christensen, L.O., Morita, H., Sinkjaer, T. \& Nielsen, J. 1998a. Evidence that a transcortical pathway contributes to stretch reflexes in the tibialis anterior muscle in man. I Physiol 512, 267-276.

Petersen, N., Christensen, L.O.D. \& Nielsen, J.B. 1998b. The effect of transcranial magnetic stimulation on the soleus $\mathrm{H}$ reflex during human walking. $J$ Physiol (Lond) 513, 599-610.

Puttemans, V., Wenderoth, N. \& Swinnen, S.P. 2005. Changes in brain activation during the acquisition of a multifrequency bimanual coordination task: from the cognitive stage to advanced levels of automaticity. $J$ Neurosci $25,4270-4278$.

Rudomin, P., Nunez, R. \& Madrid, J. 1975. Modulation of synaptic effectiveness of la and descending fibers in cat spinal cord. J Neurophysiol 38, 1181-1195.

Runge, C.F., Shupert, C.L., Horak, F.B. \& Zajac, F.E. 1999. Ankle and hip postural strategies defined by joint torques. Gait Posture 10, 161-170.

Schneider, C. \& Capaday, C. 2003. Progressive adaptation of the soleus $\mathrm{H}$-reflex with daily training at walking backward. $J$ Neurophysiol 89, 648-656.

Schubert, M., Curt, A., Jensen, L. \& Dietz, V. 1997. Corticospinal input in human gait: modulation of magnetically evoked motor responses. Exp Brain Res 115, 234 246.

Sinkjaer, T., Andersen, J.B., Nielsen, J.F. \& Hansen, H.J. 1999. Soleus long-latency stretch reflexes during walking in healthy and spastic humans. Clin Neurophysiol 110, 951-959. 
Solopova, I.A., Kazennikov, O.V., Deniskina, N.B., Levik, Y.S. \& Ivanenko, Y.P. 2003. Postural instability enhances motor responses to transcranial magnetic stimulation in humans. Neurosci Lett 337, 25-28.

Taube, W., Schubert, M., Gruber, M., Beck, S., Faist, M. \& Gollhofer, A. 2006. Direct corticospinal pathways contribute to neuromuscular control of perturbed stance. J Appl Physiol 101, 420-429.

Toft, E., Sinkjaer, T. \& Andreassen, S. 1989. Mechanical and electromyographic responses to stretch of the human anterior tibial muscle at different levels of contraction. Exp Brain Res 74, 213-219.

Trimble, M.H. \& Koceja, D.M. 1994. Modulation of the triceps surae H-reflex with training. Int J Neurosci 76, 293-303.

Trimble, M.H. \& Koceja, D.M. 2001. Effect of a reduced base of support in standing and balance training on the soleus H-reflex. Int J Neurosci 106, 1-20.
Ugawa, Y., Terao, Y., Hanajima, R., Sakai, K. \& Kanazawa, I. 1995. Facilitatory effect of tonic voluntary contraction on responses to motor cortex stimulation. Electroencephalogr Clin Neurophysiol 97, 451-454.

Verhagen, E.A., Van der Beek, A.J., Bouter, L.M., Bahr, R.M. \& Van Mechelen, W. 2004. A one season prospective cohort study of volleyball injuries. $\mathrm{Br} J$ Sports Med 38, 477-481.

Voigt, M., Chelli, F. \& Frigo, C. 1998. Changes in the excitability of soleus muscle short latency stretch reflexes during human hopping after 4 weeks of hopping training. Eur $J$ Appl Physiol Occup Physiol 78, 522-532.

Wolpaw, J.R. \& Tennissen, A.M. 2001. Activity-dependent spinal cord plasticity in health and disease. Annu Rev Neurosci $24,807-843$.

Zehr, E.P. 2002. Considerations for use of the Hoffmann reflex in exercise studies. Eur J Appl Pbysiol 86, 455-468. 